\title{
Análise das Mudanças de Parâmetros Biofísicos Sobre o Nordeste Brasileiro de 2002 a 2011 com Dados Modis
}

\author{
Argemiro L. Araújo ${ }^{1}$, Madson T. Silva ${ }^{1}$, Bernardo B. da Silva ${ }^{1}$, Carlos Antonio Costa dos Santos ${ }^{1}$, \\ Milena P. Dantas ${ }^{1}$ \\ ${ }^{1}$ Universidade Federal de Campina Grande, Unidade Acadêmica de Ciências Atmosféricas, \\ Campina Grande, PB, Brasil.
}

Recebido em: 21 de Dezembro de 2016 - Aceito em: 10 de Novembro de 2017

\begin{abstract}
Resumo
Este estudo avaliou as mudanças ocorridas no NEB por meio da análise espaço-temporal do albedo, NDVI e Ts obtidas com base no sensor MODIS no período de 2002 a 2011. Os dados obtidos por sensoriamento remoto foram associados a dados de precipitação obtidos por técnicas de reanálise para avaliação das áreas de interesse e seus respectivos pontos de amostras selecionados. O albedo apresentou mais de $75 \%$ de seus dados na faixa de 0,10 a 0,20 e os valores de Ts foram superiores a $28{ }^{\circ} \mathrm{C}$ em $88 \%$ dos pontos. As análises do NDVI e da precipitação apontaram que $73 \%$ e $76 \%$ dos dados foram superiores a 0,50 e a $1900 \mathrm{~mm} / \mathrm{ano}$, respectivamente. Foram observados aumentos significativos de Ts em anos que mais de $75 \%$ dos dados de precipitação estiveram abaixo dos $1500 \mathrm{~mm} /$ ano e crescimento de NDVI associado a anos de aumento da precipitação. O teste de Mann-Kendall ao nível de 5\% apontou a ocorrência de tendências significativas apenas para os alvos nas áreas de caatinga e cerrado. Os maiores valores de NDVI se situaram em áreas de clima tropical úmido e de clima equatorial, com valores acima de 0,70 .
\end{abstract}

Palavras-chave: Albedo, NDVI, temperatura da superfície, precipitação, sensoriamento remoto.

\section{Analysis of Biophysical Parameters Changes Over Northeastern Brazil From 2002 to 2011 With Modis Data}

\begin{abstract}
This study evaluated the changes occurring in the NEB through the time-space analysis of albedo, NDVI and Ts obtained with MODIS data in the period from 2002 to 2011 . The data collected by remote sensing were associated with precipitation data obtained by reanalysis to evaluate the areas of interest and their respectivCensoe selected sample points. The albedo presented more than $75 \%$ of its data in the range of 0.10 to 0.20 and Ts values were higher than $28^{\circ} \mathrm{C}$ in $88 \%$ of the points. The NDVI and precipitation analyze indicated that $73 \%$ and $76 \%$ of the data were higher than 0.50 and $1900 \mathrm{~mm} /$ year, respectively. Significant increases in Ts were observed in years that more than $75 \%$ of precipitation data were below $1500 \mathrm{~mm} /$ year and NDVI growth associated with years of precipitation increase. The Mann-Kendall test at the $5 \%$ level pointed to the occurrence of significant trends only for the targets in the caatinga and cerrado areas. The highest values of NDVI were in areas of humid tropical climate and equatorial climate, with values above 0.70 .
\end{abstract}

Keywords: Albedo, NDVI, surface temperature, rainfall, remote sensing.

\section{Introdução}

O estudo dos parâmetros biofísicos da superfície da terra, tais como o índice de vegetação por diferenca normalizada (NDVI), o albedo $(\alpha)$ e a temperatura da superfície (Ts),, nos permite quantificar e identificar padrões de mudanças ambientais e/ou climáticas, sejam eles de origem natural ou antrópica. As causas naturais da degradação do solo estão relacionadas a exposição a eventos climáticos extremos e persistentes (aridez, secas prolongadas), enquanto as causas antrópicas estão relacionadas ao

Autor de correspondência: Carlos Antonio Costa dos Santos, carlos.santos@ufcg.edu.br. 
uso não-sustentável do solo (desmatamento, agropecuária) e a fatores socioeconômicos (preços de mercado, instabilidade política) (UNCCD, 2012).

As variações desses parâmetros ao longo do tempo podem estar associadas tanto a processos de desertificação quanto a processos de recuperação da vegetação de uma área em particular (Eckert et al., 2015). Regiões semiáridas, onde as altas temperaturas, os eventos de seca e as mudanças no regime pluvial são mais acentuadas, estão mais propícias a mudanças significativas em seus sistemas físicos e biológicos (Wei et al., 2016). Neste sentido, o monitoramento de áreas tendenciadas a sofrerem impactos extremos em sua cobertura vegetal torna-se de fundamental importância para a identificação das causas responsáveis e para a solução dos problemas encontrados.

$\mathrm{O}$ sensoriamento remoto se apresenta como ferramenta fundamental para monitoramento dessas regiões, visto que sensores orbitais podem cobrir uma extensa área da superfície com uma escala temporal satisfatória, permitindo a estimativa de parâmetros que só seriam obtidos em aplicações ambientais especificas em experimento de campo (Santos et al., 2015; Silva et al., 2015). O sensor MODIS, encontrado a bordo das plataformas Terra e Aqua, se apresenta como uma poderosa ferramenta para aplicações ambientais, visto que oferece uma ampla gama de produtos disponíveis em uma escala de tempo diária com cobertura global e resoluções espaciais a partir de $250 \mathrm{~m}$. Assim, sua importância é fundamental para aplicações ambientais em grandes áreas do globo que não dispõem de informações de superfície suficientes, tais qual o nordeste brasileiro (NEB) (Ali et al., 2013; Santos et al., 2014).

O NEB apresenta uma predominância do clima semiárido na porção central da região, com temperaturas elevadas, chuvas irregulares e ocorrências de estiagens prolongadas, e algumas áreas de clima tropical na faixa litorânea, com a presença de estações secas e chuvosas bem definidas, e de clima equatorial úmido na porção noroeste, com temperaturas elevadas e chuvas abundantes (Lucena e Stosic, 2013). A região semiárida foi delimitada com base em alguns critérios definidos pelo IBGE (Instituto Brasileiro de Geografia e Estatística), tais quais: média pluviométrica anual inferior a $800 \mathrm{~mm}$, índice de aridez de até 0,5 e risco de seca maior que $60 \%$ para os municípios situados na região (IBGE, 2005). A vegetação predominante é a caatinga, porém observa-se a presença de áreas com florestas tropicais e cerrado em algumas regiões, bem como áreas com grande influência antrópica. O último censo agropecuário realizado em 2006 apontou um aumento de $30 \%$ nas áreas de lavouras no NEB, principalmente em decorrência da substituição das áreas de vegetação natural por lavouras e pastagens (IBGE, 2006).

Mudanças na cobertura vegetal são uma das respostas dinâmicas mais dominantes observadas nos ecossistemas terrestres relacionados a mudanças climáticas
(Richardson et al., 2013; Silva et al., 2016; Silva et al., 2018). O NDVI tem sido amplamente utilizado para avaliação da cobertura vegetal em diversos estudos com intuito de analisar a dinâmica da vegetação e, consequentemente, permitir a identificação de padrões de mudanças ambientais (Aquino et al., 2008; Alves et al., 2014; Silva et al., 2014). Quando aliado à técnica de sensoriamento remoto, o NDVI se torna fundamental no manejo em escala regional, principalmente em áreas bastante heterogêneas tal qual o NEB (Bezerra et al., 2014).

Outra variável bastante susceptível a mudanças ambientais é o $\alpha$, que está diretamente relacionado ao tipo de solo e cobertura vegetal. É um parâmetro chave para controle das trocas de energia nas interações superfícieatmosfera que varia espacialmente de acordo com as condições de iluminação solar, com o ciclo vegetativo e com as atividades antrópicas (He et al., 2012). Variações no albedo estão diretamente relacionadas a perda de cobertura vegetal, podendo ter aplicação direta na identificação de áreas propícias à desertificação. Geralmente o aumento dessa variável está relacionado ao aumento das áreas desmatadas devido a presença de solo exposto. Contudo, observa-se o inverso em casos de recuperação da vegetação e em épocas em que o solo se encontra mais úmido (Matos et al., 2013).

A Ts obtida a partir da banda termal dos sensores orbitais pode ser aplicada em diversos estudos, tais quais detecção de queimadas e focos de incêndios e detecção de ilhas de calor. Quando associado ao NDVI e ao albedo permite avaliar o nível de preservação ou degradação de ecossistemas ambientais e o impacto em secas severas (Araújo et al., 2012). Os sensores termais permitem a aquisição de informações para monitoramento de grandes extensões a um baixo custo quando comparados a instrumentos convencionais. A associação de dados de Ts obtidos por sensoriamento remoto a informações climáticas e morfológicas permite quantificar a resposta da vegetação em relação ao solo e outros alvos da superfície com grande confiabilidade (Fernandes et al., 2016).

Este estudo objetiva avaliar as mudanças ocorridas no NEB por meio da análise espaço-temporal do NDVI, albedo e temperatura da superfície obtidos por meio do sensor MODIS no período de 2002 a 2011.

\section{Material e Métodos}

\section{1. Área de estudo}

Segundo dados do IBGE (Censo 2010) o NEB possui uma população de 53.081.510 habitantes distribuída em uma área de aproximadamente $1.558 .196 \mathrm{~km}^{2}$. Apresenta uma predominância do clima tropical semiárido com médias anuais variando de $20^{\circ} \mathrm{C}$ em áreas mais elevadas, tais como a Chapada Diamantina, a $28{ }^{\circ} \mathrm{C}$ em áreas do litoral e do interior do semiárido. A região é caracterizada 
por um regime de chuvas bastante irregular, o que implica algumas áreas apresentarem precipitação pluviométrica média de 300 milímetros por ano, as quais ocorrem durante no máximo três meses, dando vazão a grandes períodos de estiagem. (Araújo, 2014).

Para avaliação da série de dados de NDVI, albedo e $\mathrm{T}_{\mathrm{S}}$ fez-se a extração dos pontos representativos selecionados de todas as áreas correspondentes a cada uma das AOI's descritas anteriormente. Com isto tornou-se possível a análise dos valores mínimos, médios e máximos mensais e anuais para o período de estudo. As AOI's e os respectivos pontos estão representados na Fig. 1 .

O NEB apresenta quatro tipos de biomas: Amazônia, caatinga, cerrado e mata atlântica. As áreas de floresta amazônica situam-se a noroeste do estado do Maranhão, onde as chuvas abundantes e o clima equatorial úmido favorecem seu desenvolvimento. A caatinga abrange toda área central do NEB com uma área superior a $60 \%$ da região, caracterizada por uma flora peculiar adaptada ao clima semiárido. Na porção oeste do NEB localizam-se as áreas de cerrado, sob influência do clima tropical. Na faixa litorânea encontram-se as regiões de mata atlântica, situadas em áreas de clima tropical úmido (SFB, 2016).

Para o presente estudo foram selecionadas 12 AOI's correspondentes a diferentes tipos de vegetação e áreas antropizadas obtidas com base no mapa de vegetação do Brasil (IBGE - 2002), disponível no site do Ministério do Meio Ambiente (www.mma.gov.br). A Tabela 1 descreve as AOI's escolhidas para o estudo, que representam os

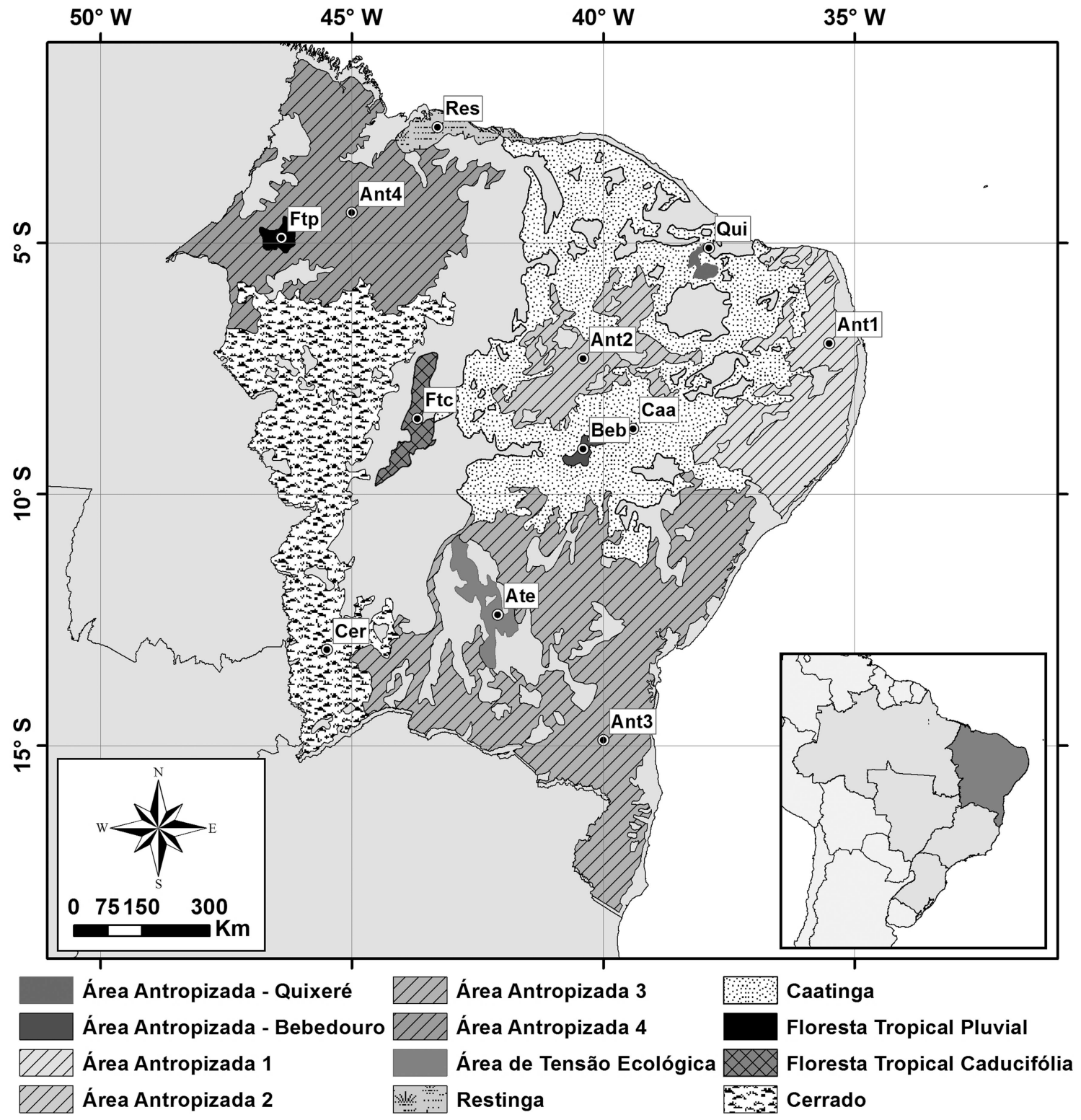

Figura 1 - Representação da Região Nordeste e das áreas de estudo selecionadas. 
Tabel 1 - Descrição das áreas de interesse e dos respectivos pontos.

\begin{tabular}{|c|c|c|}
\hline Cobertura do solo & Sigla & Descrição \\
\hline Bebedouro & Beb & $\begin{array}{l}\text { Área antropizada no interior de } \\
\text { Pernambuco }\end{array}$ \\
\hline Quixeré & Qui & Área antropizada do Ceará \\
\hline Área Antropizada 1 & Ant1 & $\begin{array}{l}\text { Rio Grande do Norte, Paraíba, Pernam- } \\
\text { buco e Alagoas }\end{array}$ \\
\hline Área Antropizada 2 & Ant2 & $\begin{array}{l}\text { Semiárido nordestino do Piauí, Ceará e } \\
\text { Pernambuco }\end{array}$ \\
\hline Área Antropizada 3 & Ant3 & Grande parte da Bahia e do Sergipe \\
\hline Área Antropizada 4 & Ant4 & Maranhão \\
\hline $\begin{array}{l}\text { Área de Tensão } \\
\text { Ecológica }\end{array}$ & Ate & Interior da Bahia \\
\hline Restinga & Res & Litoral norte do Maranhão \\
\hline Caatinga & Caa & Maior parte do semiárido nordestino \\
\hline $\begin{array}{l}\text { Floresta Tropical } \\
\text { Pluvial }\end{array}$ & Ftp & Interior do Maranhão \\
\hline $\begin{array}{l}\text { Floresta Tropical } \\
\text { Caducifólia }\end{array}$ & Ftc & Interior do Piauí \\
\hline Cerrado & Cer & $\begin{array}{l}\text { Oeste da Bahia e sul do Maranhão e do } \\
\text { Piauí }\end{array}$ \\
\hline
\end{tabular}

tipos de vegetação predominantes no NEB e áreas importantes da região.

\subsection{Obtenção do NDVI, $\alpha$ e $T_{S}$}

Foram utilizados dados do produto de reflectância MOD09A1 e MOD11A2 do período de 01 de janeiro de 2002 a 31 de dezembro de 2011 adquiridos junto à plataforma Reverb (reverb.echo.nasa.gov) disponibilizada pela agência EOSDIS (Earth Observing System Data and Information System) pertencente à NASA. Para a formação do mosaico de imagens que contém o NEB foram utilizados os Tiles h13v09, h13v10, h14v09 e h14v10.

O produto MOD09A1 fornece uma estimativa da reflectância espectral da superfície como se fosse medida ao nível do solo, considerando ausência de dispersão atmosférica ou absorção. Os dados de baixos níveis possuem correções para efeitos de gases e aerossóis atmosféricos, gerando uma base Level-2 para vários produtos de escalas maiores. É constituído de bandas com $500 \mathrm{~m}$ de resolução espacial combinadas durante 8 dias com projeção sinusoidal. Cada pixel do produto contém a melhor observação possível do período selecionado com base em alta cobertura observacional, baixo ângulo de visada, remoção de nuvens ou sombra de nuvens e aerossóis (Araújo, 2014). A Tabela 2 apresenta as bandas utilizadas no estudo.

Os dados MOD11A2 diários são obtidos com resolução espacial de $1 \mathrm{~km}$ pelo algoritmo split-window e em grades de $6 \mathrm{~km}$ pelo algoritmo day/night. No algoritmo split-window, as emissividades das bandas de 31 e 32 são estimadas a partir de tipos de cobertura do solo, da coluna de vapor de água atmosférico e da temperatura do ar na
Tabel 2 - Descrição das bandas do produto MOD09A1.

\begin{tabular}{lllc}
\hline Banda & \multicolumn{1}{c}{ Descrição } & Unidades & Fator de Escala \\
\hline 1 & Reflectância $(620-670 \mathrm{~nm})$ & Reflectância & 0,001 \\
2 & Reflectância $(841-876 \mathrm{~nm})$ & Reflectância & 0,001 \\
3 & Reflectância $(459-479 \mathrm{~nm})$ & Reflectância & 0,001 \\
4 & Reflectância $(545-565 \mathrm{~nm})$ & Reflectância & 0,001 \\
5 & Reflectância $(1230-1250 \mathrm{~nm})$ & Reflectância & 0,001 \\
6 & Reflectância $(1628-1652 \mathrm{~nm})$ & Reflectância & 0,001 \\
7 & Reflectância $(2105-2155 \mathrm{~nm})$ & Reflectância & 0,001 \\
9 & Ângulo zenital solar & Graus & 0,01 \\
11 & Ângulo azimutal relative & Graus & 0,01 \\
13 & Dia do Ano & Dia Juliano & - \\
\hline
\end{tabular}

camada mais baixa. No algoritmo de day/night, os valores diurnos e noturnos de LST e emissividade de superfície são obtidos a partir de pares de observações do MODIS durante o dia e a noite em sete bandas na faixa do infravermelho termal. O dados empregados possuem dados globais de temperatura da superfície e emissividade combinados durante 8 dias com resolução espacial de $1 \mathrm{~km} \mathrm{a}$ partir do produto MOD11A1, que por sua vez são gravados em grades de $1 \mathrm{~km}$ com projeção sinusoidal como médias dos valores para dias de céu claro durante o período de estudo (Araújo, 2014). As bandas utilizadas são descritas na Tabela 3.

O NDVI é dado por uma relação entre a reflectividade das bandas 1 e 2 do Terra/MODIS, que correspondem às bandas do vermelho e do infravermelho próximo, respectivamente:

$$
N D V I=\frac{\rho_{t, 2}-\rho_{t, 1}}{\rho_{t, 2}+\rho_{t, 1}}
$$

O albedo foi calculado a partir da integração das reflectâncias das bandas utilizando uma função de ponderação. $\mathrm{O}$ produto MOD09A1 fornece os valores da reflectância à superfície os quais podem ser aplicados na relação:

$$
\alpha=\sum_{b=1}^{n}\left[\rho_{s, b} W_{b}\right]
$$

Tabel 3 - Descrição das bandas do produto MOD11A2.

\begin{tabular}{llcc}
\hline Banda & \multicolumn{1}{c}{ Descrição } & Unidades & Fator de Escala \\
\hline 1 & $\begin{array}{l}\text { Temperatura da Superfície } \\
\text { diurna }\end{array}$ & Kelvin & $0,02 * \mathrm{~B} 1$ \\
5 & $\begin{array}{l}\text { Temperatura da Superfície } \\
\text { noturna }\end{array}$ & Kelvin & $0,02 * \mathrm{~B} 2$ \\
9 & Emissividade Band 31 & - & $0,002 * \mathrm{~B} 9+0,49$ \\
10 & Emissividade Band 32 & - & $0,002 * \mathrm{~B} 10+$ \\
11 & Dias de céu claro & - & 0,49 \\
12 & Noites de céu claro & - & - \\
\hline & & & -
\end{tabular}


com $W_{b}=$ coeficiente de ponderação (Tabela 4), que representa a fração da radiação solar que ocorre na faixa espectral da referida banda $b$.

A temperatura da superfície foi obtida diretamente a partir do produto MOD11A2 pela multiplicação da banda 1 pelo fator de escala 0,02 . Já a emissividade foi obtida pela média das bandas 9 e 10 do mesmo produto citado, após conversão pelo fator de escala.

Para a manipulação dos dados empregados no estudo, foi realizada a extração das variáveis para pontos de grades com espaçamento equidistante de $10 \mathrm{Km}$. Estes dados foram submetidos a um refinamento para exclusão de valores incoerentes e cálculo dos valores médios mensais e anuais. A partir dos dados médios foram realizadas analises estatísticas para avaliação dos resultados. Para análise das séries temporais mensais foram utilizados os pontos de amostra situados em cada uma das AOI's conforme apresentado na Fig. 1.

\section{Resultados e Discussão}

Analisando o comportamento do albedo médio representado na Fig. 2 observa-se que os maiores valores foram encontrados no oeste do estado do Maranhão e na faixa litorânea do NEB. O histograma aponta uma predominância de valores de reflectância na faixa de 0,15 a 0,20 ; que representaram mais de $75 \%$ dos pontos de grade analisados. Os valores superiores a 0,20 corresponderam a $16 \%$ dos dados, enquanto os valores abaixo de 0,15 representaram aproximadamente $9 \%$.

Os valores de Ts mostrados na Fig. 3 apresentaram uma tendência inversa aos dados de albedo, com destaque para valores médios abaixo de $27{ }^{\circ} \mathrm{C}$ no noroeste do Maranhão e no litoral sul da Bahia. As áreas do interior da região, com predominância do bioma caatinga, e a porção oeste, com predominância do cerrado, apresentaram os maiores valores de Ts, com medias superiores a $30{ }^{\circ} \mathrm{C}$. O histograma de frequências revelou que aproximadamente $54 \%$ dos dados foram superiores a $30{ }^{\circ} \mathrm{C}$. Os valores abaixo de $28{ }^{\circ} \mathrm{C}$ corresponderam a $12 \%$ dos dados, enquanto os valores situados na faixa de $28{ }^{\circ} \mathrm{C}$ a $30{ }^{\circ} \mathrm{C}$ apontam para $34 \%$.

De acordo com a Fig. 4, os menores valores de NDVI foram observados na porção central do Nordeste, que corresponde a região de clima semiárido, com valores inferiores a 0,42 . Os maiores valores foram encontrados no oeste e no litoral sul da Bahia e em áreas dos estados do Maranhão e do Piauí, com dados superiores a 0,54. O

Tabel 4 - Valores da constante $W_{b}$ para o sensor MODIS (Tasumi et al., 2008).

\begin{tabular}{llllllll}
\hline Coeficiente & \multicolumn{7}{c}{ Banda } \\
\hline & 1 & 2 & 3 & 4 & 5 & 6 & 7 \\
$W_{b}$ & 0,215 & 0,215 & 0,242 & 0,129 & 0,101 & 0,062 & 0,036 \\
\hline
\end{tabular}

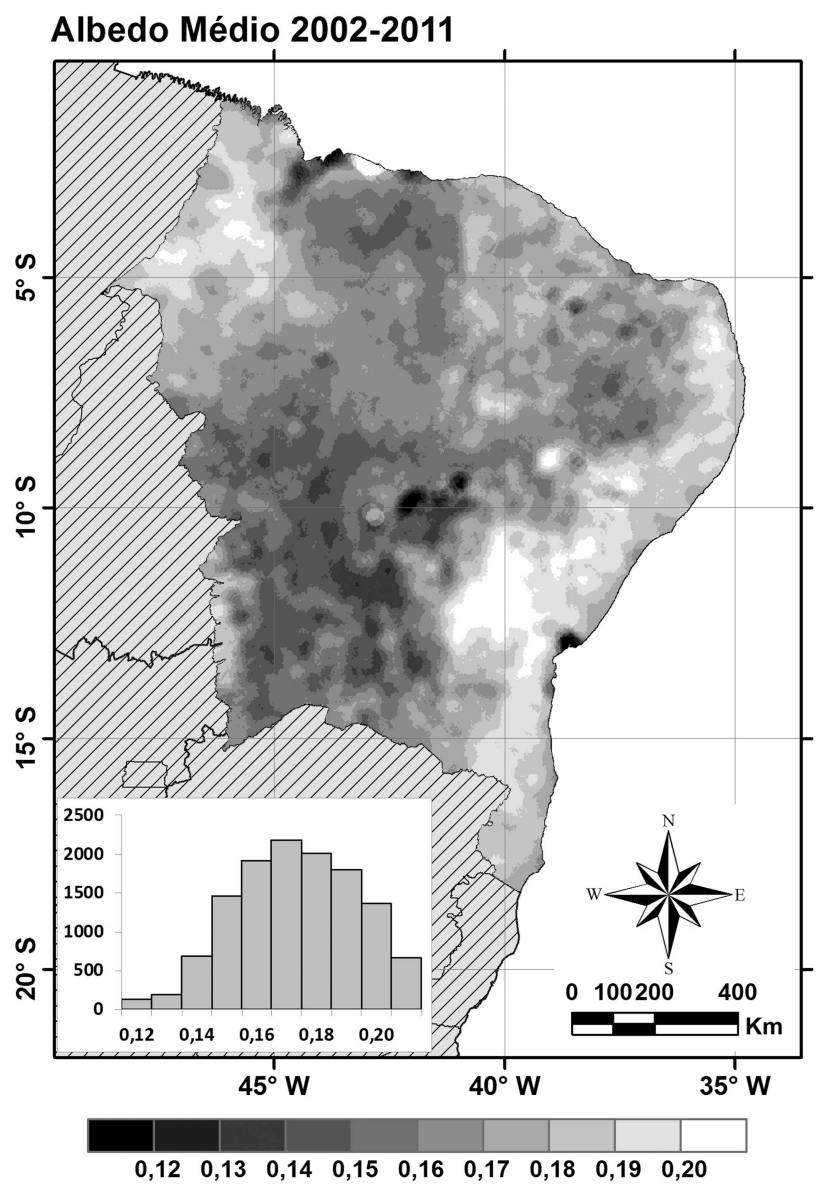

Figura 2 - Valores médios de albedo para a Região Nordeste no período de 2002 a 2011.

histograma aponta que aproximadamente $51 \%$ dos dados se encontram na faixa de 0,50 a 0,62 . Os valores inferiores a 0,50 e superiores a 0,62 corresponderam a $27 \%$ e $22 \%$, respectivamente.

Para verificação dos padrões espaciais das variáveis biofísicas obtidas neste estudo foram utilizados dados suplementares de precipitação desenvolvidos e disponibilizados segundo Xavier et al. (2015). Os dados interpolados estão representados na Fig. 5. Os maiores valores de precipitação, superiores a $1900 \mathrm{~mm}$ anuais, foram encontrados no noroeste do Maranhão e na faixa litorânea do NEB. Os menores valores, inferiores a $1100 \mathrm{~mm}$, foram observados na porção central da região, correspondentes a aproximadamente $32 \%$ dos dados. O histograma mostrou uma predominância de valores na faixa de 1100 a $1900 \mathrm{~mm}$, apontando $57 \%$ dos dados. Os valores superiores a 1900 representaram apenas $19 \%$ dos pontos de grade.

Os comportamentos anuais das variáveis para todos os pontos de grade do NEB podem ser observados na Fig. 6. Contata-se que mais de $50 \%$ dos dados de albedo situaram-se na faixa de 0,15 a 0,20 em todos os anos analisados. Sua mediana permaneceu praticamente invariável 


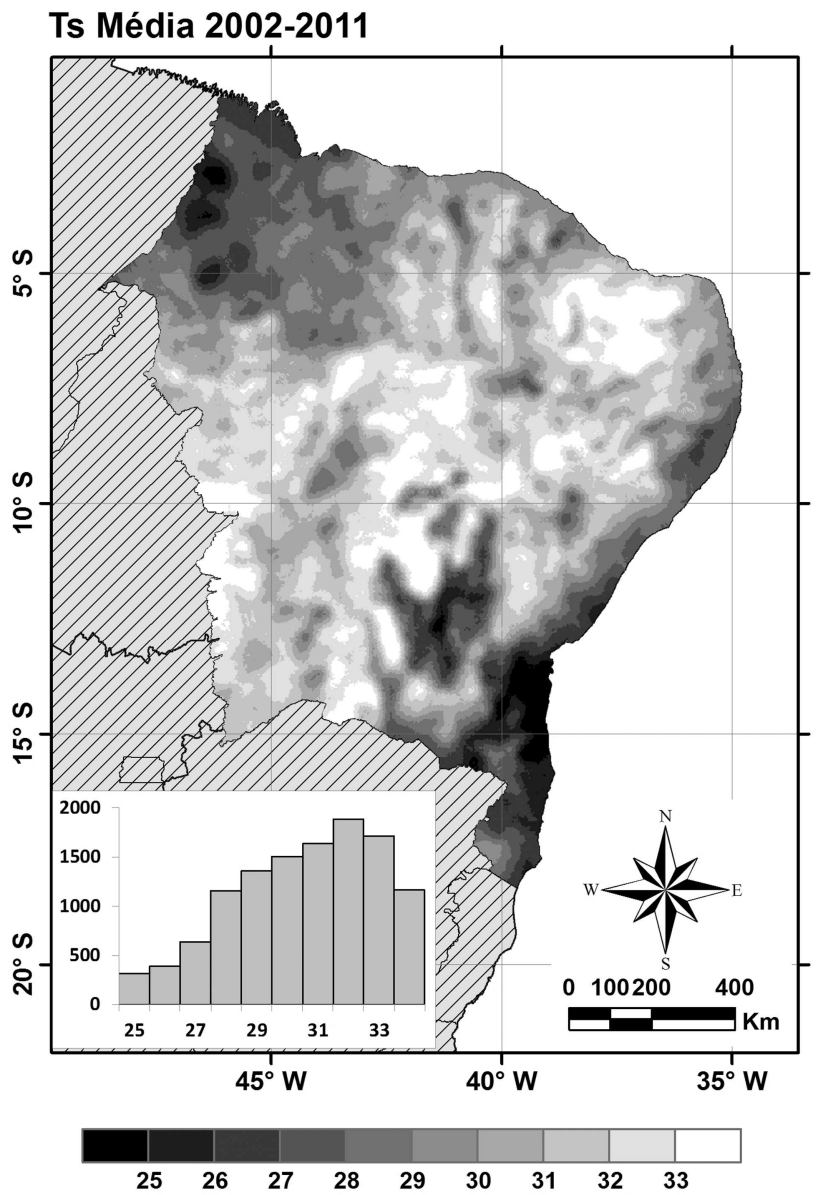

Figura 3 - Valores médios de Ts $\left({ }^{\circ} \mathrm{C}\right)$ para a Região Nordeste no período de 2002 a 2011.

no período de 2002 a 2008, apresentando uma queda nos anos de 2009 e 2010, provavelmente relacionada ao aumento da precipitação observado em 2009. O teste de Tukey mostrou que os anos de 2002, 2003, 2004 e 2006 não apresentaram diferença significativa em suas médias ao nível de $5 \%$ de significância. O mesmo foi observado para os pares de anos 2005-2007 e 2006-2008. Todos os demais anos apresentaram diferença significativa entre si.

O NDVI apresentou um ligeiro crescimento de sua mediana no período de 2002 a 2006, que pode estar relacionado ao aumento da precipitação nos anos de 2004 a 2006. Observa-se uma ligeira queda nos valores no ano de 2007, o que condiz com as quedas nos valores de precipitação para o mesmo período. Os anos seguintes apresentaram uma tendência positiva do NDVI, diretamente relacionada ao aumento da precipitação em 2008 e 2009. Mesmo com a diminuição da precipitação em 2010 contata-se uma manutenção dos valores de NDVI nos anos de 2009 a 2011. A faixa de NDVI de 0,4 a 0,6 apresentou uma predominância de mais de $50 \%$ dos dados analisados em todos os anos. O teste de Tukey ao nível de 5\% revelou

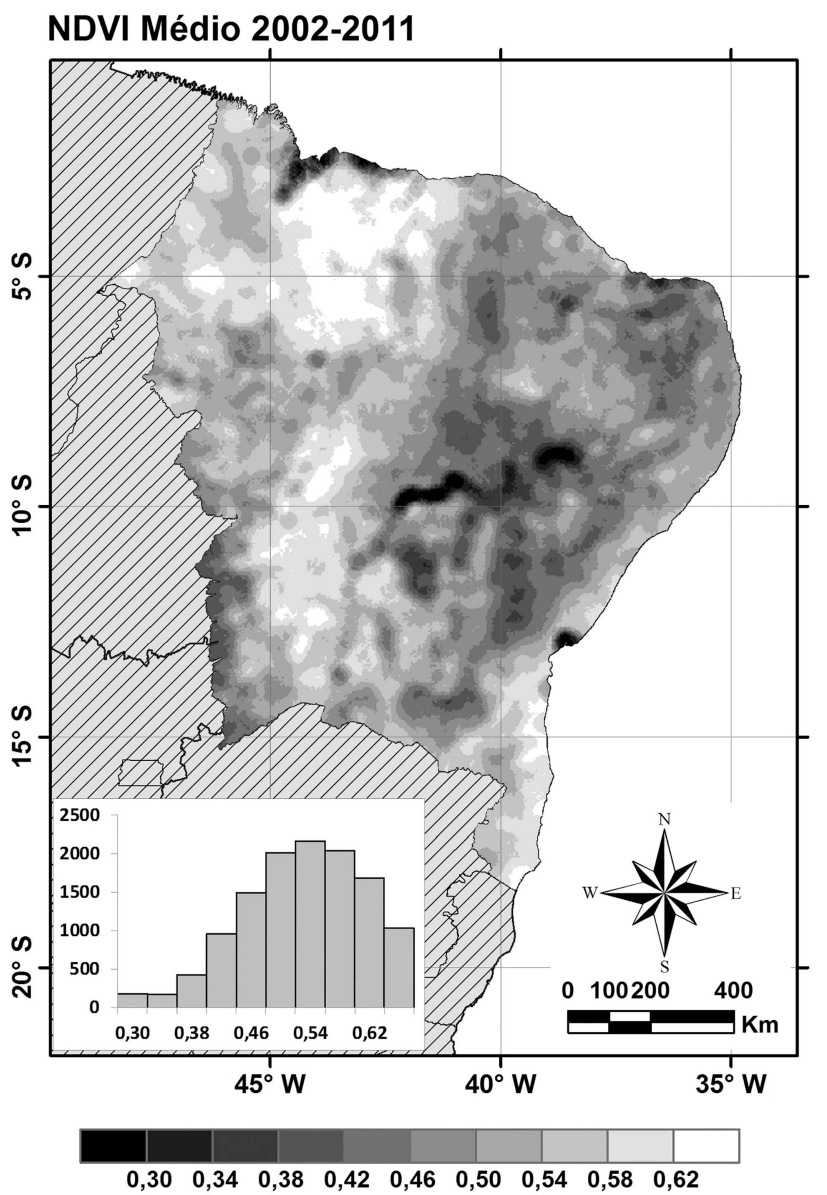

Figura 4 - Valores médios de NDVI para a Região Nordeste no período de 2002 a 2011.

semelhança significativa apenas para 6 pares de anos, conforme exposto na Fig. 6.

Os perfis anuais de Ts apresentaram um comportamento inverso aos perfis de precipitação. Observa-se um decréscimo no período de 2002 a 2006, de 2007 a 2009 e de 2010 a 2011. Nos anos em que mais de $75 \%$ dos dados de precipitação estiveram abaixo dos $1500 \mathrm{~mm}$ foram constatados aumentos significativos nos valores de Ts, mais precisamente nos anos de 2002, 2003, 2007 e 2010. Para estes anos citados observou-se que as medianas dos dados de Ts foram superiores a $30{ }^{\circ} \mathrm{C}$, bem diferente dos demais anos onde $50 \%$ dos valores estavam abaixo dos $30{ }^{\circ} \mathrm{C}$. O teste de Tukey ao nível de 5\% apontou semelhança de médias entre os anos de 2002, 2005 e 2010, o que se mostra fortemente relacionado aos anos de estiagem. Os anos mais chuvosos também influenciaram uma semelhança estatística entre dois pares de dados de Ts: 2005-2008 e 2009-2011.

O teste de Kruskal-Wallis constatou que não houve diferença significativa entre as amostras de medianas dos anos estudados para os dados de albedo, NDVI e Ts. 


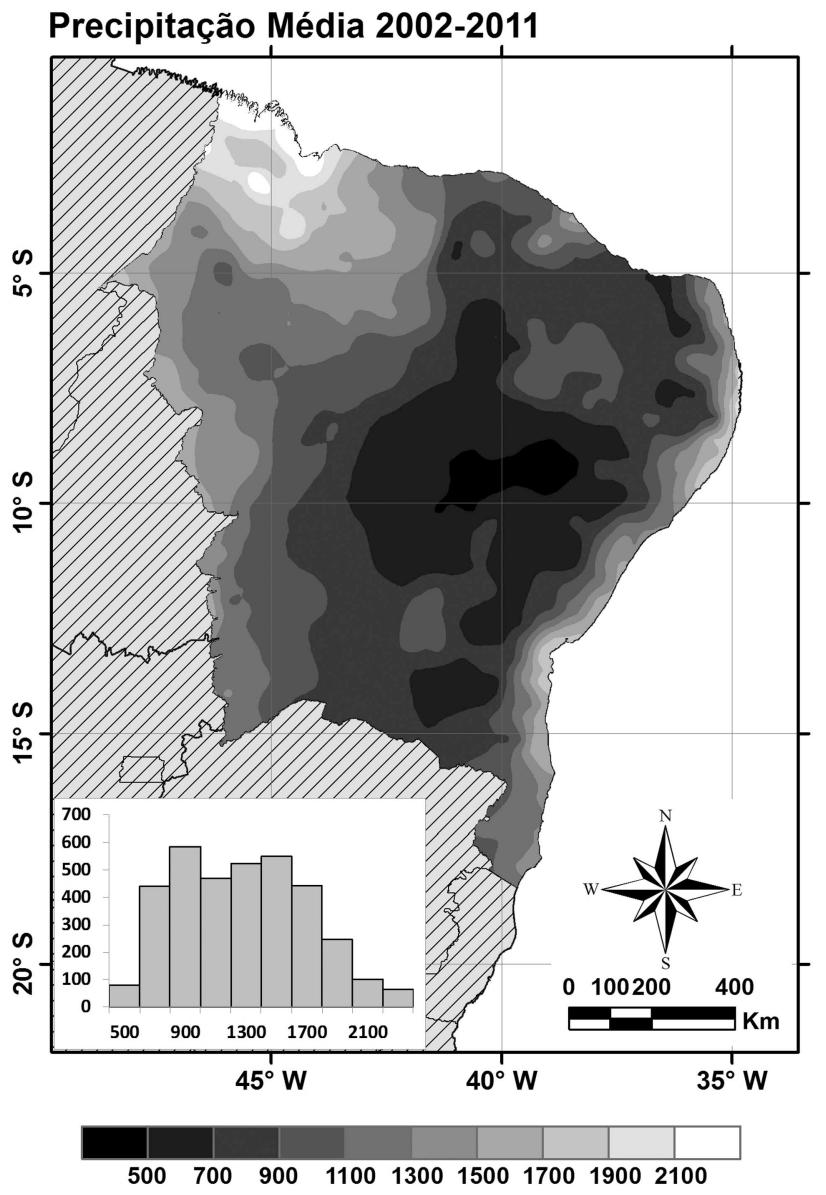

Figura 5 - Valores médios de precipitação $(\mathrm{mm})$ para a Região Nordeste no período de 2002 a 2011.

Contudo, houve diferença significativa para os dados de precipitação.

Os dados amostrais de cada AOI foram submetidos ao teste de normalidade de Shapiro-Wilk. Conforme exposto na Tabela 5, as áreas Ant1, Ant2 e Ant3 não se adequaram a uma distribuição normal para o albedo. As amostras Cer e Ant3 também não seguiram uma distribuição normal, neste caso para os dados de NDVI. Em todos os outros casos abordados o comportamento das amostras seguiu uma distribuição normal.

Os 12 alvos selecionados em cada um dos AOI's também foram submetidos ao teste de tendência de MannKendall para avaliação da presença ou ausência de tendência na série de dados estudada considerando o efeito de sazonalidade natural dos dados. Conforme exposto na Tabela 6, os alvos Ant2 e Caa apresentaram uma tendência de aumento do NDVI e da precipitação e de redução do albedo e da Ts. O alvo Cer demonstrou um comportamento inverso, indicando aumento nos valores de albedo e Ts e redução nos valores de NDVI. O alvo Beb apresentou tendência negativa para Ts, positiva para o NDVI e nula para as demais variáveis. Observou-se uma tendência negativa de albedo e Ts para o alvo Ftc não seguida pelas
Tabela 5 - Teste de normalidade de Shapiro-Wilk ao nível de 5\% de significância para os pontos de amostras de dados de cada AOI. Com (-) distribuição normal e (+) distribuição não-normal.

\begin{tabular}{lcccc}
\hline Ponto & Albedo & Ts & NDVI & Prec. \\
\hline Ant1 & + & - & - & - \\
Ant2 & + & - & - & - \\
Ant3 & + & - & + & - \\
Ant4 & - & - & - & - \\
Beb & - & - & - & - \\
Qui & - & - & - & - \\
Ate & - & - & - & - \\
Caa & - & - & - & - \\
Cer & - & - & + & - \\
Res & - & - & - & - \\
Ftc & - & - & - & - \\
Ftp & - & + & - & - \\
\hline
\end{tabular}

Tabel 6 - Teste de tendência de Mann-Kendall ao nível de 5\% de significância para os pontos de amostras de dados de cada AOI. Com (-) tendência negativa, $(0)$ tendência nula e $(+)$ tendência positiva.

\begin{tabular}{lcccc}
\hline Ponto & Albedo & Ts & NDVI & Prec. \\
\hline Ant1 & 0 & - & 0 & 0 \\
Ant2 & - & - & + & + \\
Ant3 & 0 & + & 0 & 0 \\
Ant4 & - & 0 & 0 & 0 \\
Beb & 0 & - & + & 0 \\
Qui & - & 0 & 0 & 0 \\
Ate & 0 & 0 & 0 & 0
\end{tabular}

\begin{tabular}{lcccc} 
Caa & - & - & + & + \\
Cer & + & + & - & 0 \\
Res & 0 & 0 & 0 & 0 \\
Ftc & - & - & 0 & 0 \\
Ftp & 0 & 0 & 0 & 0 \\
\hline
\end{tabular}

demais variáveis. Os demais alvos apontaram um comportamento estacionário para o período abordado no estudo.

A Tabela 7 apresenta os dados estatísticos das variáveis biofísicas abordadas no estudo para cada área de interesse selecionada. As maiores variações de albedo foram observadas nas áreas Ant3, Caa e Res, que apresentaram amplitudes superiores a 0,17. Estas áreas apresentaram os valores máximos superiores a 0,24 e mínimos inferiores a 0,11 . O mesmo se aplica para os dados de NDVI, onde se observa mínimos inferiores a 0,22 e máximos superiores a 0,71 para as mesmas áreas descritas. $\mathrm{O}$ valor máximo de 0,30 foi observado na área Res, esta que também apresentou o maior desvio-padrão. 

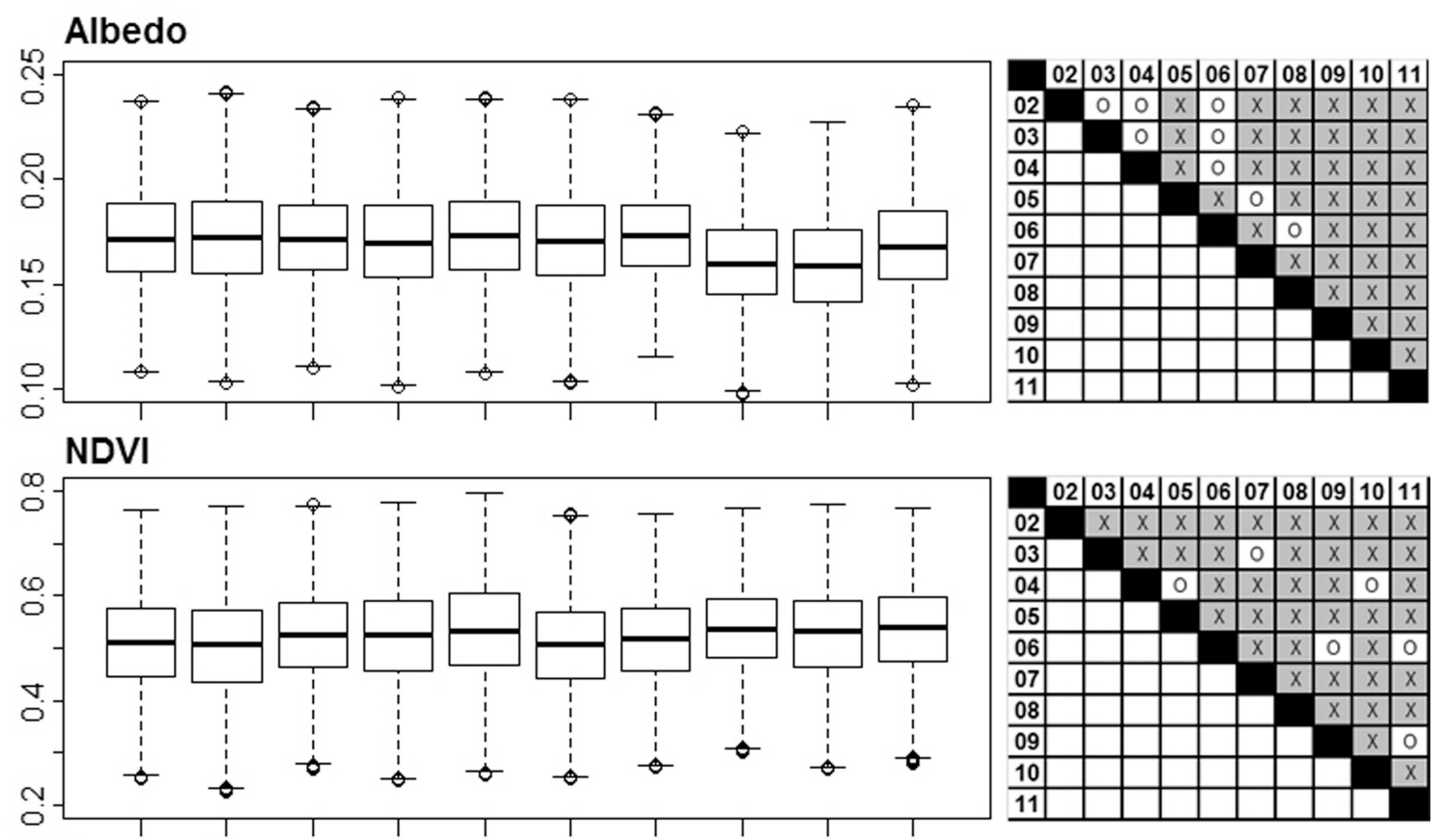

\section{Temperatura}
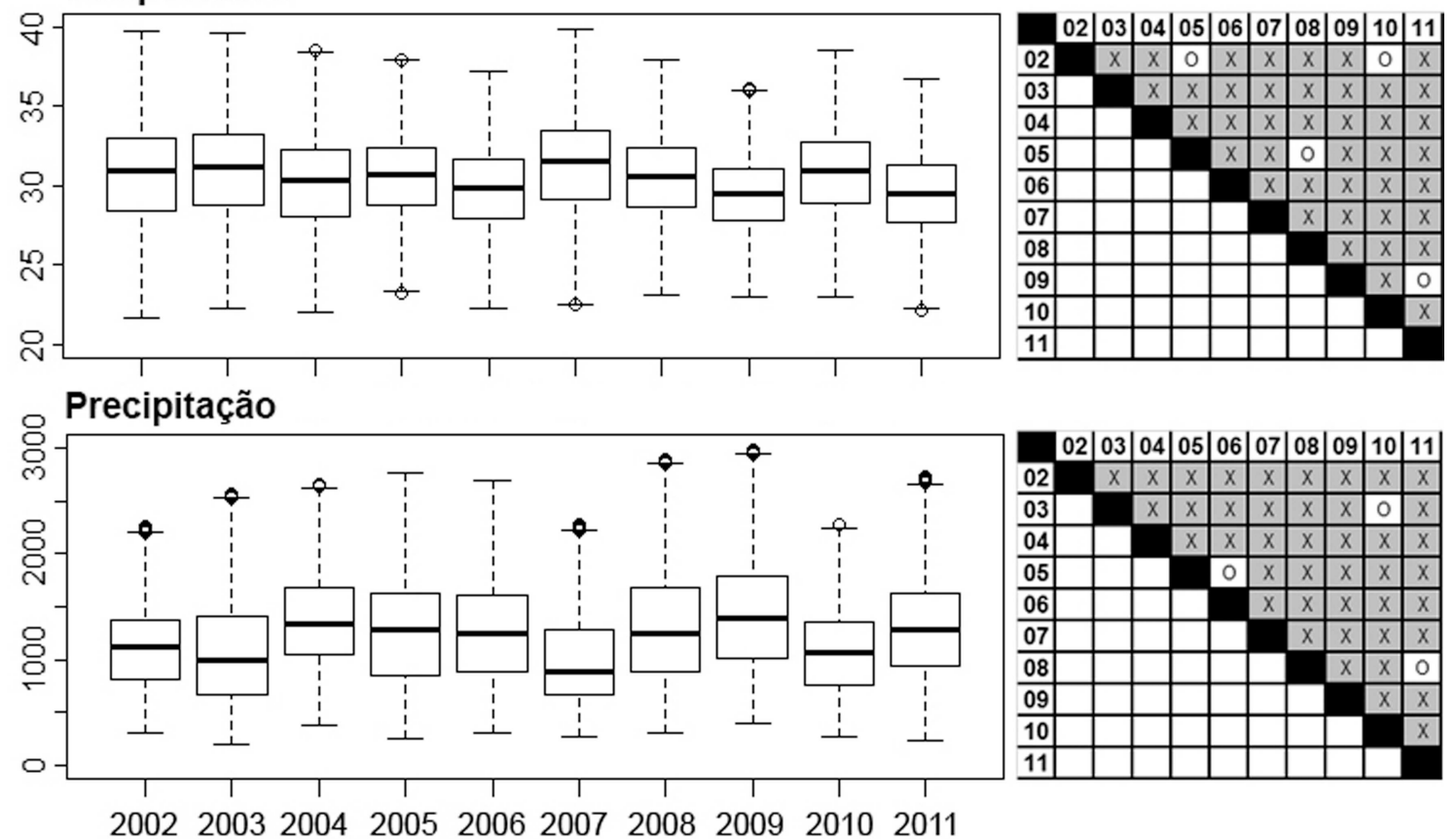

Figura 6 - Média anual dos dados de Albedo, NDVI, Ts $\left({ }^{\circ} \mathrm{C}\right)$ e Precipitação $(\mathrm{mm})$ no período de 2002 a 2011 para os pontos de grade do NEB e teste de Tukey ( $\mathrm{X}=$ diferenças significativas ao nível de $5 \%$ entre os anos).

Os maiores valores de NDVI ocorreram em áreas de clima tropical úmido (Ant3) e em áreas de clima equatorial (Ant4, Ftp e Res) com valores máximos superiores a 0,70 . Valores inferiores a 0,15 ocorreram nas áreas Ant3 e Caa, assim como os maiores valores de desvio-padrão. Dentre as áreas de floresta tropical contata-se que a Ftp apresenta valores de NDVI superiores a Ftc. A área Ftp também apresentou valores de Ts inferiores aos da área Ftc, com diferenças que variaram de 2 a $6{ }^{\circ} \mathrm{C}$. O cerrado apresentou valores de NDVI ligeiramente superiores aos da caatinga, enquanto que os dados de Ts se mostraram similares nas duas regiões. As áreas antropizadas apresentaram as maiores amplitudes de temperatura, com valores superiores a $10^{\circ} \mathrm{C}$. 
Tabel 7 - Dados estatísticos de albedo, NDVI e Ts $\left({ }^{\circ} \mathrm{C}\right)$ para as áreas de interesse no período de 2002 a 2011.

\begin{tabular}{|c|c|c|c|c|c|c|c|c|c|c|c|c|}
\hline & \multicolumn{4}{|c|}{ Albedo } & \multicolumn{4}{|c|}{ NDVI } & \multicolumn{4}{|c|}{ Ts } \\
\hline & MAX & MED & MIN & DEV & MAX & MED & MIN & DEV & MAX & MED & MIN & DEV \\
\hline Ant1 & 0,23 & 0,18 & 0,12 & 0,01 & 0,63 & 0,47 & 0,29 & 0,05 & 37,32 & 30,14 & 25,44 & 1,94 \\
\hline Ant2 & 0,21 & 0,17 & 0,13 & 0,01 & 0,63 & 0,47 & 0,33 & 0,05 & 35,98 & 31,61 & 25,37 & 1,51 \\
\hline Ant3 & 0,24 & 0,18 & $\mathbf{0 , 0 7}$ & 0,02 & 0,73 & 0,51 & 0,14 & 0,07 & 37,52 & 29,08 & 22,60 & 2,80 \\
\hline Ant4 & 0,23 & 0,18 & 0,13 & 0,02 & 0,76 & 0,59 & 0,28 & 0,05 & 34,62 & 28,14 & 24,34 & 1,02 \\
\hline Beb & 0,18 & 0,16 & 0,13 & 0,01 & 0,50 & 0,43 & 0,31 & 0,02 & 34,55 & 32,08 & 28,44 & 0,93 \\
\hline Qui & 0,20 & 0,17 & 0,14 & 0,01 & 0,62 & 0,48 & 0,37 & 0,02 & 36,28 & 33,63 & 29,60 & 0,49 \\
\hline Ate & 0,19 & 0,15 & 0,11 & 0,01 & 0,65 & 0,54 & 0,39 & 0,04 & 37,37 & 30,26 & 25,14 & 2,51 \\
\hline $\mathrm{Caa}$ & 0,24 & 0,17 & 0,08 & 0,01 & 0,68 & 0,46 & 0,15 & 0,07 & 38,00 & 31,82 & 25,49 & 1,59 \\
\hline Cer & 0,21 & 0,16 & 0,11 & 0,01 & 0,69 & 0,51 & 0,28 & 0,05 & 36,55 & 31,69 & 26,22 & 1,31 \\
\hline Res & 0,30 & 0,18 & 0,11 & $\mathbf{0 , 0 3}$ & 0,71 & 0,51 & 0,22 & 0,09 & 32,37 & 29,13 & 26,39 & 0,92 \\
\hline Ftc & 0,18 & 0,15 & 0,12 & 0,01 & 0,69 & 0,58 & 0,44 & 0,05 & 35,75 & 31,25 & 26,67 & 1,58 \\
\hline Ftp & 0,21 & 0,18 & 0,15 & 0,01 & 0,70 & 0,62 & 0,53 & 0,03 & 29,74 & 26,64 & 24,65 & 0,86 \\
\hline
\end{tabular}

Alves et al. (2014) encontraram valores mínimos e máximos de NDVI de 0,22 e 0,53 para a região do Alto Curso do Rio Paraíba - PB, respectivamente. Também encontraram valores médios de albedo da ordem de 0,15 para a mesma área de estudo no ano de 2013. Bezerra et al. (2014) obtiveram valores de albedo variando de 0,10 a $0,20 \mathrm{em}$ áreas de caatinga, valores médios de NDVI variando de 0,25 a 0,66 e de Ts de 18 a $31{ }^{\circ} \mathrm{C}$ para a região do Parque Nacional da Furna Feia - RN no período de 2007 a 2010. Matos et al. (2013) determinaram valores de Ts variando de 20 a $31{ }^{\circ} \mathrm{C}$ para a Bacia do Rio Pajeú - PE nos anos de 2001 e 2009.

\section{Conclusões}

O comportamento médio das variáveis para o período de 2002 a 2011 demonstrou que mais de $75 \%$ dos dados de albedo se situaram na faixa de 0,10 a 0,20 e que os dados de Ts superiores a $28{ }^{\circ} \mathrm{C}$ representaram $88 \%$ dos dados avaliados. As áreas de caatinga e cerrado apresentaram médias de Ts superiores a $30^{\circ} \mathrm{C}$. A porção central do NEB apresentou valores de NDVI inferiores a 0,42 e de precipitação inferiores a $1100 \mathrm{~mm} / \mathrm{ano}$, que correspondem a $27 \%$ e $32 \%$ dos dados avaliados, respectivamente.

Analisando os perfis médios anuais das variáveis observou-se que mais de $50 \%$ dos dados de albedo e de NDVI se situaram nas faixas de 0,15 a 0,20 e de 0,40 a 0,60 ; respectivamente. Foi constatada correlação entre os períodos de crescimento do NDVI e do aumento da precipitação de 2004 a 2006 e de 2008 a 2009. Foram observados aumentos significativos de Ts nos anos em que mais de $75 \%$ dos dados de precipitação estiveram abaixo dos $1500 \mathrm{~mm}$ anuais. Nestes anos a mediana dos dados de Ts deslocou-se para valores superiores a $30{ }^{\circ} \mathrm{C}$ em contraposição aos demais anos, em que esteve abaixo desse valor.

O teste de Tukey ao nível de $5 \%$ de significância demonstrou que a grande maioria dos pares de anos comparados apresentou diferença significativa entre si para todas as variáveis abordadas. Já o teste de Kruskal-Wallis apontou diferença significativa para as amostras de medianas apenas para os dados de precipitação. $\mathrm{O}$ teste de normalidade de Shapiro-Wilk identificou que a maioria dos conjuntos de dados seguiu uma distribuição normal. O teste de Mann-Kendall ao nível de 5\% apontou que os alvos Ant2 e Caa apresentaram tendência negativa para os dados de albedo e Ts e tendência positiva para os dados de NDVI e precipitação. O alvo Cer apresentou comportamento inverso. Os demais alvos não apresentaram tendência significativas e foram classificados como estacionários.

As análises de cada AOI identificaram que os maiores valores de NDVI se situaram em áreas de clima tropical úmido (Ant3) e de clima equatorial (Ant4, Ftp, Res) com valores superiores a 0,70 . Os menores valores foram observados em áreas de caatinga. Em geral, as áreas antropizadas apresentaram amplitudes de Ts superiores a $10{ }^{\circ} \mathrm{C}$. As áreas Ant3, Caa e Res apresentaram as maiores amplitudes de albedo, com valores superiores a $0,24 \mathrm{e}$ inferiores a 0,17 .

\section{Agradecimentos}

Os autores agradecem ao Conselho Nacional de Desenvolvimento Científico e Tecnológico (CNPq), pelo apoio financeiro a esta pesquisa (Processo $\mathrm{N}^{\circ} .446172 / 2015-4$ ) e 
pela concessão das bolsas de Produtividade em pesquisa aos autores (Processo N. 301348/2015-4), a Coordenação de Aperfeiçoamento de Pessoal de Nível Superior - Brasil (CAPES) - Finance Code 001 (Projeto Pró-Alertas - processo N ${ }^{\circ}$. 88887.091737/2014-01; e Bolsa de Professor Visitante no Exterior Júnior - processo $\mathrm{N}^{\circ}$. 88881.172029/2018-01), além da concessão das bolsas de estudo. Adicionalmente, agradecemos à Campina Geotech Consultoria em Meteorologia Ltda. pela parceria na elaboração do artigo.

\section{Referências}

ALI, A.; BIE, C.A.J.M.; SKIDMORE, A.K.; SCARROTT, R.G.; HAMAD, A.; VENUS, V.; LYMBERAKIS, P. Mapping land cover gradients through analysis of hyper-temporal NDVI imagery. International Journal of Applied Earth Observation and Geoinformation, v. 23, p. 301-312, 2013.

ALVES, T.L.B.; AZEVEDO, P.V.; SANTOS, C.A.C.; SANTOS, F.A.C. Comportamento sazonal do albedo e dos índices de vegetação na bacia hidrográfica do Alto Curso do Rio Paraíba. Revista Brasileira de Geografia Física, v. 7, p. 1015-1027, 2014.

AQUINO, C.M.S.; ALMEIDA, J.A.P.; OLIVEIRA, J.G.B. Estudo da cobertura vegetal/uso de terra nos anos de $1987 \mathrm{e}$ 2007 no núcleo de degradação/desertificação de São Raimundo Nonato - Piauí. RA'E GA, v. 25, p. 252-278, 2012.

ARAÚJO, A.L. SANTOS, C.A.C.; SILVA, B.B.; BEZERRA, B. G.; BORGES, V.P. Refinamento de imagens termais do Landsat 5 - TM com base em classes de NDVI. Revista Brasileira de Meteorologia, v. 27, p. 484-490, 2012.

ARAÚJO, A.L. Operacionalização do balanço de energia e evapotranspiração em escala regional com dados de sensores orbitais. 101f. Tese (Doutorado em Meteorologia). Universidade Federal de Campina Grande, Campina Grande, Paraíba, 2014.

SILVA, E.F.F. Parâmetros biofísicos obtidos por sensoriamento remoto em região semiárida do estado do Rio Grande do Norte, Brasil. Revista Brasileira de Engenharia Agrícola e Ambiental, v. 18, p. 73-84, 2014.

ECKERT, S.; HÜSLER, F.; LINIGER, H.; HODEL, E. Trend analysis of MODIS NDVI time series for detecting land degradation and regeneration in Mongolia. Journal of Arid Environments, v. 133, p. 16-28, 2015.

FERNANDES, A.C.G.; COUTINHO, M.A.N.; SANTOS, V.G.; NASCIMENTO, C.R. Utilização de intervalos de índices de vegetação e temperature da superfície para detecção de queimadas. Caderno de Ciências Agrárias, v. 8, p. 30-40, 2016.

HE, T.; LIANG, S.; WANG, D.; WU, H.; YU, Y.; WANG, J. Estimation of surface albedo and directional reflectance from Moderate Resolution Imaging Spectroradiometer (MODIS) observations. Remote Sensing of Environment, v. 199, p. 286-300, 2012.
INSTITUTO BRASILEIRO DE GEOGRAFIA E ESTATÍSTICA (IBGE). Cadastro de Municípios localizados na Região Semiárida do Brasil - 2005. Disponível em: http://www.ibge.gov.br.

INSTITUTO BRASILEIRO DE GEOGRAFIA E ESTATÍSTICA (IBGE). Censo agropecuário - 2006: Brasil, Grandes Regiões e Unidades da Federação. Rio de Janeiro, 2006. p. 775. Disponível em: http://www.ibge.gov. br.

LUCENA, L.R.R.; STOSIC, T. Temperatura do nordeste brasileiro via análise de lacunaridade. Sigmae, v. 2, p. 76-80, 2013.

MATOS, R.C.M.; CANDEIAS, A.L.B.; TAVARES-JUNIOR, J. R. Mapeamento da vegetação, temperatura e albedo da bacia hidragráfica do Pajeú com imagens MODIS. Revista Brasileira de Cartografia, v. 65, p. 139-160, 2013.

RICHARDSON, A.D.; KEENAN, T.F.; MIGLIAVACCA, M.; RYU, Y.; SONNENTAG, O.; TOOMEY, M. Climate change, phenology, and phenological control of vegetation feedbacks to the climate system. Agricultural and Forest Meteorology, v. 169, p. 156-173, 2013.

SANTOS, C.A.C.; WANDERLEY, R.L.N., ARAÚJO, A.L., BEZERRA, B.G. Obtenção do saldo de radiação em áreas de pastagem e floresta na Amazônia (estação seca) através do sensor MODIS. Revista Brasileira de Meteorologia, v. 29, p. 420-432, 2014.

SANTOS, F.A.C.; SANTOS, C.A.C.; SILVA, B.B.; ARAÚJO, A.L.; CUNHA, J.E.B.L. Desempenho de metodologias para estimativa do saldo de radiação a partir de imagens MODIS. Revista Brasileira de Meteorologia, v. 30, p. 295-306, 2015.

SERVIÇO FLORESTAL BRASILEIRO (SFB). Recursos Florestais - Os biomas e suas florestas. Disponível em: http://www.florestal.gov.br. (acesso em 07.10.2016).

SILVA, M.T.; SILVA, V.P.R.; SOUZA, E.P. Morphometric analysis of the basin low middle São Francisco river. Journal of Hyperspectral Remote Sensing, v. 4, n. 8, p. 168-174, 2014.

SILVA, M.T.; SILVA, V.P.R; SOUZA, E. P.; ARAÚJO, A.L. SWAT model application to estimate the flow in the basin of lower-middle São Francisco River. Revista Brasileira de Geografia Física, v. 8, p. 1615-1627, 2015.

SILVA, V.P.R.; SILVA, M.T.; SOUZA, E.P. Influence of land use change on sediment yield: a case study of the sub-middle of the São Francisco River basin. Engenharia Agrícola, v. 36, n. 6, p. 1005-1015, 2016.

SILVA, V.P.R.; SILVA, M.T, SINGH, V.P, SOUZA, E.P, BRAGA, C.C, HOLANDA R.M.; BRAGA, A.C.R. Simulation of stream flow and hydrological response to landcover changes in a tropical river basin. CATENA, v. 162, p.166-176, 2018.

TASUMI, M.; ALLEN, R. G.; TREZZA, R. At-surface reflectance and albedo from satellite for operational calculation of land surface energy balance. Journal of Hydrologic Engineering, v. 13, p. 51-63, 2008.

UNCCD, 2012. United Nations Convention to Combat Desertification. www.unced.int (acesso em 05.10.2016)

WEI, S.; DE-YONG, Y.; ZHONG-PING, S.; JUN-GE, Z.; XIAO-XUAN, L.; QIAN, L. Vegetation changes in the 
agricultural-pastoral areas of northern China from 2001 to

2013. Journal of Integrative Agriculture, v. 15, p. $1145-1156,2016$.
This is an Open Access article distributed under the terms of the Creative Commons Attribution Non-Commercial License which permits unrestricted non-commercial use, distribution, and reproduction in any medium provided the original work is properly cited. 